\title{
A new constant-pressure molecular dynamics method for finite system
}

\author{
D. Y. Sun \\ Institute of Solid State Physics, Academia Sinica, 230031-Hefei, P. R. China \\ X. G. Gong \\ Institute of Solid State Physics, Academia Sinica, 230031-Hefei, P. R. China \\ Department of Physics, Fudan University, Shanghai 200433, P.R. China
}

(Received October 23, 2018)

\begin{abstract}
In this letter, by writing the volume as a function of coordinates of atoms, we present a new constantpressure molecular dynamics method with parameters free. This method is specially appropriate for the finite system in which the periodic boundary condition does not exist. Simulations on the carbon nanotube and the Ni nanoparticle clearly demonstrate the validity of the method. By using this method, one can easily obtain the equation of states for the finite system under the external pressure.
\end{abstract}

PACS numbers: $02.70 . \mathrm{Ns}, 61.46+\mathrm{w}, 36.40 . \mathrm{Ei}$

The molecular dynamics (MD) simulation method is a powerful tool, widely used in chemistry, physics, and materials science. [1] As a very important achievement, constant-pressure MD proposed by Andersen, [2] and subsequently extended by Parrinello and Rahman, 3] has provided a powerful method to study systems under the external pressure. Now the constant-pressure MD has become a standard tool to study the physical properties under the external pressure. Especially, it has played a central role in investigating the structural phase transition as well as in geophysical and astrophysical applications.

Recently, the study of low-dimensional and biological system under the external pressure become a considerable interest. [4.5] In particular, studies on the carbon nanotube, [6] clusters and nanocrystal, such as CdSe, CdS and Si nanocrsystals, [7] have revealed a wealth of interesting new phenomena. Usually computer simulation could substantially complement the experimental information. However, the traditional constant-pressure MD method was designed for the infinite system with a periodic boundary condition, it can not be directly applied to the finite system, such as nanotubes and nanocrystals, in which the periodic boundary condition does not exist. For this reason it is necessary to develop a new computational scheme to study the finite system. Very recently, Martonak, Molteni and Parrinello have successfully studied the pressure induced amorphorpization of $\mathrm{Si}_{35} \mathrm{H}_{36}$ cluster by introducing pressure-transmitting liquid. [8] In order to have a well-defined isotropic constant pressure on the cluster, the number of particles and the volume of the pressure-transmitting liquid should be much larger than that of the cluster, thus it costs a significant overhead of the computation. Additionally, one also must determine how the liquid atoms interacts with themselves and with cluster atoms so that the liquid does not crystallize, or, in the time scale of the simulation the liquid does not undergo a glass transition.

In this paper, by writing the volume as a function of coordinates of atoms, we propose a new constant-pressure $\mathrm{MD}$, which should be appropriate for finite systems with arbitrary shape. This new constant-pressure MD is parameter free, and can be used in any system with arbitrary shape, especially in nanocrystals. We will demonstrate its validity by classical simulations of a carbon nanotube, and small Ni particles, its extension to $a b-i n i t i o$ molecular dynamics method is straight forward. [9]

We write the Lagrangian $L$ of a $N$-atom system as:

$$
L=\sum_{i}^{N} \frac{\mathbf{p}_{i}^{2}}{2 m_{i}}-\left(\phi\left(\left\{\mathbf{r}_{\mathbf{i}}\right\}\right)+P_{e x t} V\right)
$$

where $\mathbf{r}_{\mathbf{i}}, m_{i}, \mathbf{p}_{i}$ are the coordinates, mass, momentum of ith atom respectively, and $\phi$ is potential of system. $V$ is the volume of the system, and $P_{e x t}$ is the external pressure.

If the system obeys the Newton's mechanism, the equations of motion for $\mathbf{r}_{i}$ derived from the Lagrangian $L$ read,

$$
\frac{d}{d t}\left(\frac{\partial L}{\partial \dot{\mathbf{r}}_{i}}\right)=\frac{\partial L}{\partial \mathbf{r}_{i}}
$$

Obviously the enthalpy will be conserved. The equations of motion derived from Eq. 2 produce the constant pressure ensemble for the system, as we show bellow. For an equilibrium system with the external pressure,

$$
\frac{1}{3 V}\left(\sum_{i}^{N} m_{i} v_{i}^{2}-\sum_{i}^{N} \mathbf{r}_{i} \cdot \nabla \phi-\sum_{i}^{N} \mathbf{r}_{i} \cdot P_{e x t} \nabla V\right)=0
$$

Then we have,

$$
\sum_{i}^{N} m_{i} v_{i}^{2}-\sum_{i}^{N} \mathbf{r}_{i} \cdot \nabla \phi=\sum_{i}^{N} \mathbf{r}_{i} \cdot P_{e x t} \nabla V
$$


It is well known that, in statistical physics, [10] the volume is an additive quantities, which can be written as a summation of the volume of individual atoms,

$$
V=\sum_{i}^{N} v_{i}
$$

where $v_{i}$ is the volume of the $i$ th atom, which can be generally written as a cubic homogeneous function of its nearest neighbor distance $r_{i j}$, i.e.

$$
v_{i}=\sum_{j \neq i} f\left(r_{i j}^{3}\right)
$$

According to Euler theorem,

$$
\sum_{i}^{N} \mathbf{r}_{i} \cdot \nabla V=3 V
$$

So from Eq. 4 we have

$$
P_{\text {ext }}=P_{\text {int }}=\frac{1}{3 V}\left(\sum_{i}^{N} m_{i} v_{i}^{2}-\sum_{i}^{N} \mathbf{r}_{i} \cdot \nabla \phi\right)
$$

where $P_{\text {int }}$ refers to the internal pressure, since the external pressure $P_{e x t}$ is a constant, $P_{i n t}$ is also a constant. Thus, by writing the volume as a function of atomic coordinates, a new constant-pressure $\mathrm{MD}$ is presented, in which no extra parameter is introduced.

The present constant-pressure MD method has several advantages. First, this method can make the calculation more realistic, without needing to choose the mass for the volume as in traditional constant-pressure MD, [2] which directly affects the time scale of the relaxation. Secondly, in the present method, the responding of system to the external pressure is more physical. This is specially important for the inhomgenous system. However, in the traditional constant-pressure MD, the responding of system to the external pressure is essentially linear, i.e., the volume of the system is linearly scaled according to the difference of the internal and external pressure.

The key to the success of the present constant-pressure MD method is to define the volume as a function of atomic coordinates. In the traditional constant-pressure MD method, the volume is a generalized coordinates, which has equal importance as an atomic coordinate, the constant pressure is dynamically achieved by directly changing the volume of system. However, in the present scheme, the constant pressure is obtained by dynamically changing the motion of each atom.

To show how well this new constant-pressure MD works in the real application, we have simulated carbon nanotubes and Ni nanocrystals under the external pressure.

In the real simulation, to get an exact formalism for the atomic volume is physically difficult. However, there are a varity of sufficient approximations. One of the simplest and direct way is based on the Wigner-Seitz premitive cell, we used the scaled volume of the atomic sphere to approximate the Wigner-Seitz premitive cell, which has the following form,

$$
v_{i}=\gamma_{i} \frac{4 \pi}{3} \sum_{j \neq i}\left(\frac{r_{i j}}{2}\right)^{3},
$$

where the summation runs over all the first nearest neighbors of the $i$ th atom, $\gamma_{i}$ is a scale factor which is closely related the number of the nearest neighbours of the $i$ th atom. At the high temperature or in the system where the first nearest neighbors frequently change, we used a more sophisticated form. [9]

The definition of the volume is not unique, our definition for Ni nanocrystals is one of them. If it is necessary, one can make more elegant one. In the study of carbon nanotube, we fix the periodic length in axial direction. The slight different definition of the volume for nanotubes will be presented else. [11]

The interaction between carbon atoms is described by the parametrized potential developed by Brenner [12] according to the Tersoff bonding formalism [13], which is widely used to study mechanical properties of carbon nanotube [14]. The Sutton-Chen potential is used to describe the interaction between $\mathrm{Ni}$ atoms, 15 which is also widely used in the literature [16]. We find that, the obtained results for the carbon nanotube and Ni nanocrystal are in good agreement with what obtained by other methods.

The evolution of the instantaneous pressure in the simulation of the $(10 \times 10)$ carbon nanotube and the $\mathrm{Ni}$ nanocrystal (Fig. 1), shows that the present method does recover a constant pressure simulation. Although the instantaneous pressure of the system fluctuates, their average values are equal to the set external pressure, 0.7 GPa for the carbon nanotube and 7.0 GPa for the Ni nanocrystal. The correlation between the volume and pressure can also be clearly observed.

What shown in Fig. 2 is the calculated energy via volume (pressure) for carbon nanotube, the close agreement between the static calculation and the present constantpressure MD simulation clearly demonstrates the validity of the present method. In the static calculation, energies are calculated with linear scaling the radius of the carbon nanotube without relaxing the atomic positions. All the energies shown in the lower panel of Fig. 2 are relative to the minimum energies, and the volume are renormalized by the equilibrium volume without the external pressure. We performed the constant-pressure MD simulation at the zero temperature and $300 \mathrm{~K}$ at various external pressure from $0 \mathrm{GPa}$ to $2 \mathrm{GPa}$. Both the static calculation and the constant-pressure MD simulation at the zero temperature give the very similar results, the slight difference comes from the relaxation of the atomic coordinates.

We show the enthalpy as a function of the reduced 
volume for static and present MD results in the middle panel of Fig. 2. It can be seen that, at each volume, the enthalpy of present MD, in which the structures under the external pressure are relaxed, is always smaller than that from the static calculation as it should be. The difference of the calculated enthalpy between the static and $\mathrm{MD}$ at $0 \mathrm{~K}$ increasing with reducing volume suggests the atomic relaxation becomes more and more important. The reasonable agreement between the ab-initio and the model potential calculation shows that the present model potential can qualitatively describe the behavior of the carbon nanotube. The new constant-pressure MD also correctly describe the finite temperature properties.

The equation of states (EOS) at finite temperature for Ni nanocrystal can be obtained through the simulation, which is reasonable in agreement with the bulk phase EOS calculated by traditional constant-pressure MD. Fig. 3 shows the equation of states for a $\mathrm{Ni}$ nanocrystal and bulk phase calculated by the new constant-pressure MD and traditional MD respectively. All the energies shown in Fig. 3 are relative to the minimum energies, and the volume is renormalized by the equilibrium volume without the external pressure at $300 \mathrm{~K}$. The nanocrystal and bulk phase show similar behavior. However, from the figure, one can see that the nanocrystal is not as hard as the bulk phase. If assuming first-order Birch-Murnaghan EOS. 18] is also appllicable to the finite system, we yields the bulk modulus $136 \mathrm{GPa}$ and $166 \mathrm{GPa}$ for nanocrystal and bulk phase respectively, in agreement with our previous results. 19]

In summary, by writing the volume as a function of coordinates of atoms, we have proposed a new constantpressure molecular dynamics method for the finite system with parameters free, where the external pressure could be exactly implemented. Simulations on the carbon nanotube and Ni nanoparticles have clearly demonstrated the validity of the method, in which the constant pressure is recovered. We have also shown that, with the newly proposed scheme, the EOS for the finite system can be calculated through a molecular dynamics simulation. The extension to $a b$ - initio molecular dynamics method is straight forward and is in progress.

We thanks Dr. D. J. Shu and Dr. G. Chen for the technical assistance. This work is supported by the National Science Foundation of China, the special funds for major state basic research and CAS projects.

[1] M. P. Allen and D. J. Tildesley, Computer Simulation of Liquid, Clarendon Press, Oxford, (1997).

[2] H. C. Andersen, J. Chem. Phys. 72, 2384 (1980).

[3] M. Parrinello and A. Rahman, J. Appl. Phys. 52, 7182

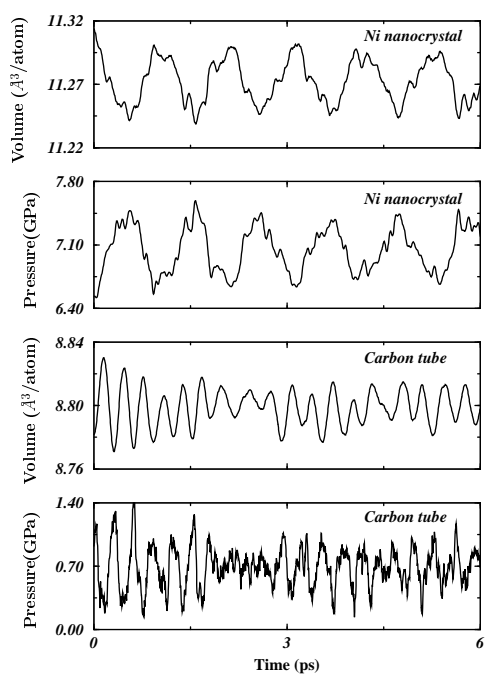

FIG. 1. The evolution of the instantaneous pressure and volume through MD runs for the carbon nanotube (lower two) and Ni nanocrystal (upper two). The pressure and volume of the system fluctuates around the average value. The new constant-pressure MD does recover a constant pressure. 

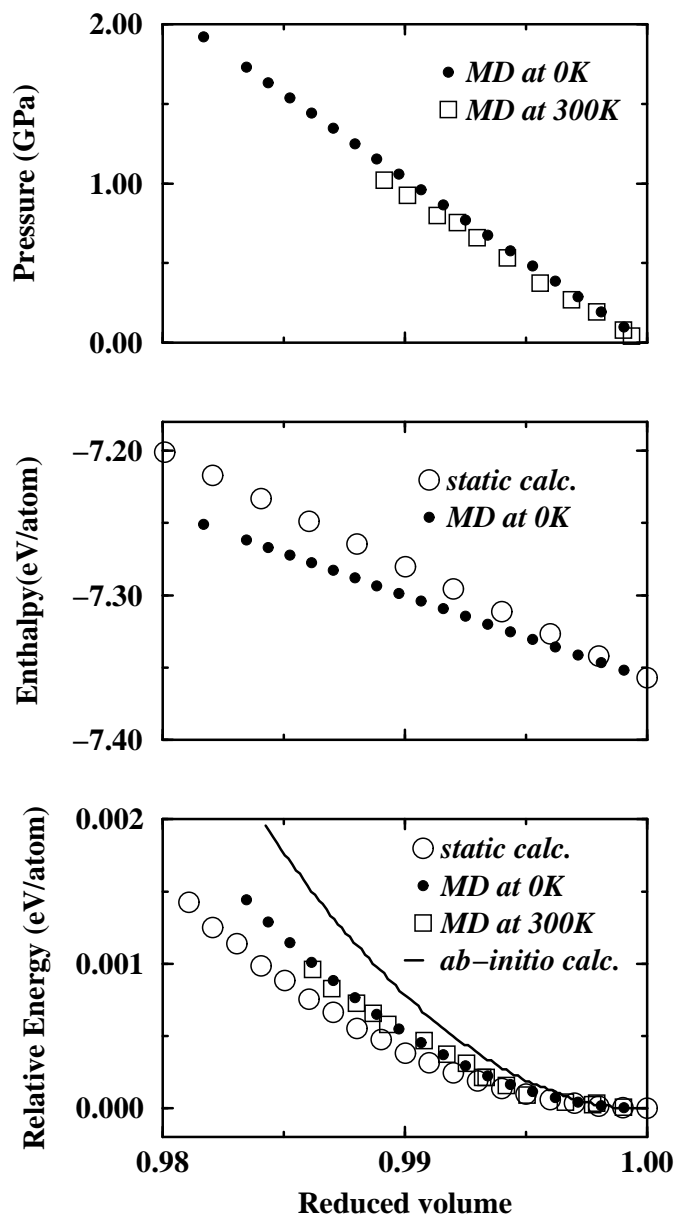

FIG. 2. The calculated properties for the carbon nanotube. Low panel: The energy as a function of the volume by the new constant-pressure MD at $0 \mathrm{~K}$ (filled circle), $300 \mathrm{~K}$ (open square), static calculation (open circle) and $a b-$ initio result (solid line). Middle panel: The enthalpy as a function of the reduced volume for static (open circle) and present MD (filled circle) calculation. Up panel: The pressure-volume relation of the carbon nanotube at $0 \mathrm{~K}$ (filled circle) and 300 $\mathrm{K}$ (open square). Our MD results at zero temperature are in good agreement with the static results except for large volume charge, where the structural relaxation includes in our MD runs.
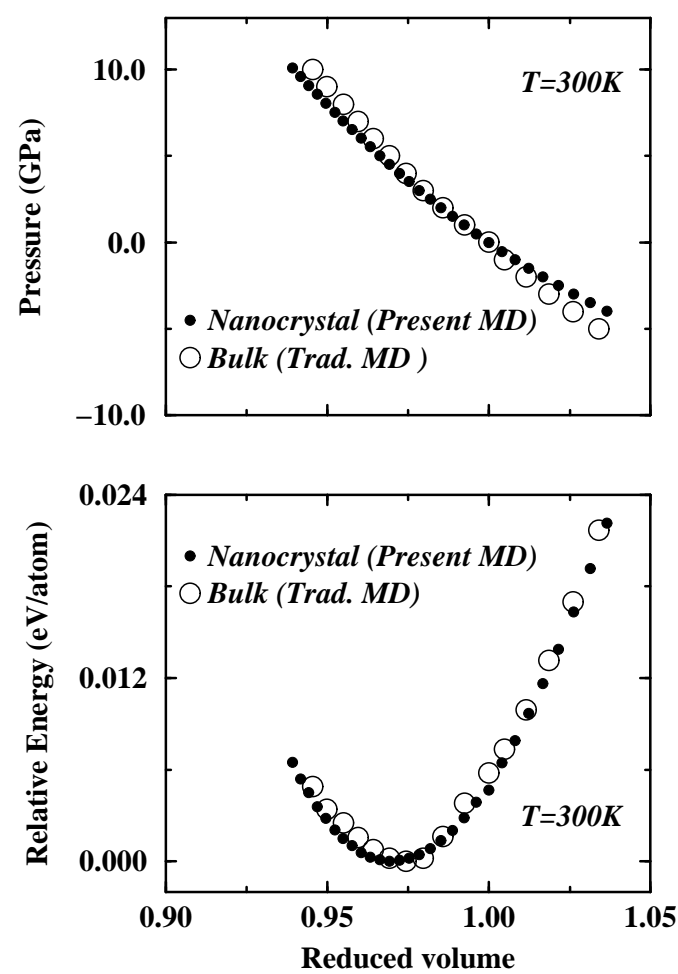

FIG. 3. The equation of states for Ni nanocrystals (filled circles) and bulk phase (open circles), where the data for $N i_{3151}$ nanocrystals and bulk phase are calculated by the new and traditional molecular dynamics simulation respectively. 
(1981);ibid Phys. Rev. Lett. 45, 1196 (1980).

[4] Proceedings of the XXXVI European High-Pressure Research Group Meeting on molecular and Low Dimensional System under pressure, Catania, Italy, 1998, edited by G. G. N. Angilella, R. Pucci, and G. Piccitto, and F. Siringo, Book of Abstracts.

[5] F. Gradrat et al., Eur. J. Biochem, 262, 900 (1999).

[6] See for example, Weidian Shen and Bin Jiang, Bao Shan Han and Si-shen Xie, Phys. Rev. Lett. 84, 3634 (2000); S. Reich, H. Jantoljak and C. homsen, Phys. Rev. B 61 , R13389 (2000); U. D. Venkateswran et al., Phys. Rev. B 59, 10928 (1999); M. J. Peters, L. E. McNeil, J. P. Lu and D. Kahn, Phys. Rev. B 61, 5939 (2000).

[7] S. H. Tolbert and A. P. Alivisatos, Z. Phys. D 26, 56 (1993); ibid. J. Chem. Phys. 102, 4642 (1995); ibid. Science 265, 373 (1994); ibid. Annu. Rev. Phys. Chem. 46, 595 (1995); S. H. Tolbert et al., Phys. Rev. Lett. 76, 4384 (1996).

[8] R. Martonak, C. Molteni and M. Parrinello, Phys. Rev. Lett. 84, 682 (2000).

[9] D. Y. Sun and X. G. Gong, to be published.

[10] Statistical Physics 3rd edition Part 1, L. D. Landau and E. M. Lifshitz, Pergamon Press, 1976.

[11] D. Y. Sun, D. J. Shu and X. G. Gong, to be published.

[12] D. W. Brenner, Phys. Rev. B 42, 9458 (1990).

[13] J. Tersoff, Phys. Rev. Lett. 61, 2879 (1988).

[14] see for example, M. B. Nardelli, B. I. Yakobson and J. Bernholc, Phys. Rev. Lett. 81, 4656 (1998); B. I. Yakobson, C. J. Brabec and J. Bernholc, Phys. Rev. Lett. 76, 2511 (1996); Yueyuan Xia, Yuchen Ma, Yuelin Xiang, Yuguang Mu, Chunyu Tan and Liangmo Mei, Phys. Rev. B 61, 11088 (2000).

[15] A. P. Sutton and J. Chen, Phil. Mag. Lett. 61, 139 (1990).

[16] see for example, Y. Xiang, D. Y. Sun and X. G. Gong, J. Phys. Chem. A 104, 2746 (2000); J. Uppenbrink and D. J. Wales, J. Chem. Phys. 96 , 8520 (1992); A. Kara and T. S. Rahman, Phys. Rev. Lett. 81, 1453 (1998).

[17] G. Chen et al., unpublised.

[18] F. Birch, J. Geophys. Res. 57, 227 (1952).

[19] D. Y. Sun, X. G. Gong and X. Q. Wang, submitted; D. Y. Sun and X. G. Gong, submitted. 